\title{
Determination of whether supplemental oxygen therapy is beneficial during exercise training in patients with COPD: A systematic review and meta-analysis
}

\author{
YING LIU ${ }^{1}$ and FANGHUA GONG ${ }^{2}$ \\ ${ }^{1}$ Fourth Department of Respiratory and Critical Care Medicine, Hunan Provincial People's Hospital \\ (The First Affiliated Hospital of Hunan Normal University); ${ }^{2}$ Department of Nursing, Hunan Provincial \\ People's Hospital (The First Affiliated Hospital of Hunan Normal University), Changsha, Hunan 410005, P.R. China
}

Received July 22, 2019; Accepted September 9, 2019

DOI: $10.3892 /$ etm.2019.8026

\begin{abstract}
Exercise training is an integral component of the pulmonary rehabilitation program for patients with chronic obstructive pulmonary disease (COPD). The aim of this study was to systematically search the published literature and analyze the evidence on the efficacy of supplemental oxygen in improving outcomes during exercise training in patients with COPD. The PubMed, Scopus, Cochrane Central Register of Controlled Trials (CENTRAL) and Google scholar databases were searched electronically for articles in the English language published up to May, 2019. In total, 7 trials were included in this systematic review and meta-analysis. There was considerable heterogeneity amongst the included studies. Meta-analysis indicated no significant difference in power (random: $\mathrm{MD}=-2.38$; 95\% CI, -5.79 to 1.03; $\mathrm{P}=0.86)$ and maximum energy expenditure $\left(\mathrm{VO}_{2} \max \right)$ (random: $\mathrm{MD}=-0.01 ; 95 \% \mathrm{CI},-0.06$ to $0.07 ; \mathrm{P}=0.45$ ) between the oxygen and control groups on maximal exercise testing. Qualitative analysis of the included studies revealed no benefits of supplemental oxygen in improving exercise capacity and dyspnea scores. Data on the quality of life assessed by the Chronic Respiratory Disease Questionnaire was pooled for 95 participants in the study group and 91 participants in the control group. The results indicated no beneficial effects of supplemental oxygen in improving quality of life outcomes (random: $\mathrm{MD}=-0.09 ; 95 \% \mathrm{CI},-0.16$ to $-0.01 ; \mathrm{P}=0.59$ ). On the whole, the findings of this study indicate that supplemental oxygen during the exercise training of patients with COPD does not improve exercise capacity, dyspnea scores and
\end{abstract}

Correspondence to: Dr Fanghua Gong, Department of Nursing, Hunan Provincial People's Hospital (The First Affiliated Hospital of Hunan Normal University), 61 Jiefang West Road, Changsha, Hunan 410005, P.R. China

E-mail: carolgfh@163.com

Key words: chronic obstructive lung disease, oxygen therapy, physical endurance, dyspnea, quality of life quality of life. However, the quality of the evidence is weak. Multi-center randomized controlled trials with homogenous methodology and intervention are required to provide stronger evidence on this subject.

\section{Introduction}

In the USA, chronic obstructive pulmonary disease (COPD) affects $7 \%$ individuals in the age group of 25-75 years and is the 4th leading cause of mortality in North America and Europe $(1,2)$. This chronic progressive disease results in the gradual loss of lung function, eventually leading to hypoxemia and dyspnea, with patients complaining of chronic cough, sputum production and shortness of breath (3). Dyspnea from air-flow limitation usually restricts patients with COPD from performing strenuous physical activities with the resultant loss of strength and the endurance of skeletal muscles (4). Exercise intolerance is a characteristic of COPD and is observed in the early stages of the disease. The management of all patients with COPD, functionally disabled by breathlessness, includes pulmonary rehabilitation (PR) $(5,6)$. An integral component of PR is physical training, which can lead to significant improvements in exercise capacity, breathlessness, fatigue and health-related quality of life outcomes of patients with COPD (7). PR benefits individuals by decreasing minute ventilation for any given external work possibly by improving the metabolic capacity of skeletal muscles (8).

Oxygen supplementation has been an ergogenic aid for patients with COPD. Long-term oxygen therapy has been shown to be beneficial for patients with chronic resting hypoxemia (9). Ambulatory oxygen has also been used for the treatment of patients who experience hypoxemia during routine daily activities, but are normoxemic at rest (10). Several clinical trials have investigated the role of supplemental oxygen during exercise training in PR programs (11-13). Patients with exercise-induced desaturation may not tolerate high-intensity exercise and may require reduced intensity training during PR, thereby limiting its effectiveness (5). It is postulated that oxygen may enhance the exercise training of patients with COPD (11). The mechanism of supplemental oxygen in increasing exercise performance has been attributed to decreased ventilatory response, possibly due to 
a blunted carotid body drive or delayed lactic acidosis (14). The results of randomized controlled trials (RCTs) however, have been conflicting $(13,15)$. A Cochrane meta-analysis in 2007 reviewing 5 RCTs, reported limited evidence for oxygen supplementation during exercise training for individuals with COPD (2). There is thus a need for an updated systematic review and meta-analysis investigating the role of oxygen therapy during exercise training for patients with COPD.

\section{Data and methods}

Selection criteria. The primary objective of this systematic review was to investigate the role of supplemental oxygen in improving outcomes following exercise training in patients with COPD. This study was conducted in line with the Preferred Reporting Items for Systematic Reviews and Meta-analyses (PRISMA) statement (16) and guidelines of the Cochrane Handbook for Systematic Reviews of Intervention (17). Only RCTs evaluating the efficacy of supplementary oxygen therapy during the exercise training of patients with COPD were included. Abstracts, non-English language studies, uncontrolled studies and retrospective studies were excluded. The detailed inclusion and exclusion criteria applied for participants, interventions and outcomes are presented in Table I.

Search strategy. PubMed, Scopus, Cochrane Central Register of Controlled Trials (CENTRAL) and Google scholar databases (first 100 results) were electronically searched for articles published up to May, 2019. Key words used in various combinations were as follows: Oxygen therapy [MeSH], oxygen [MeSH], supplemental oxygen [Free text], chronic obstructive pulmonary disease [MeSH], chronic obstructive lung disease [MeSH], chronic obstructive airway disease [MeSH], pulmonary rehabilitation [Free text], exercise [MeSH], dyspnea [MeSH], quality of life [MeSH] and physical endurance [MeSH]. References of included studies and review articles were analyzed for the identification of any additional studies.

Collection of data and analysis. Two independent reviewers examined potentially eligible studies for inclusion in the review. Following the removal of duplicates, studies were scrutinized by their title and abstracts. Full-texts of selected articles were then scanned for inclusion. Any difference in opinion was resolved by discussion. The following data from the included trials were extracted: Authors, publication year, study type, sample size, demographic data, interventions used, exercise protocol, outcomes assessed and study conclusions.

Quality assessment. The Cochrane Collaboration risk assessment tool for RCTs was used for assessing the risk of bias (18). Seven criteria were evaluated for each study: Random sequence generation, allocation concealment, blinding of participants and personnel, blinding of outcome assessment, incomplete outcome data, selective outcome reporting and other biases. The included studies were judged for each item and rated as 'high risk', 'low risk' or 'unclear risk'.

Statistical analysis. Several variable exercise tests have been used for patients with COPD, such as maximal/incremental/progressive exercise tests, constant power/sub-maximal/endurance exercise tests, functional exercise tests and shuttle walk tests. Only results from the same exercise protocol were combined. Meta-analysis was conducted only if at least 3 studies reported data on the same scale. In studies where exercise outcomes were measured on oxygen and room air, only room air values were considered. Outcome data extracted were entered into Review Manager [RevMan, version 5.3; Nordic Cochrane Centre (Cochrane Collaboration), Copenhagen, Denmark, 2014] for quantitative analysis. Changes in outcome scores were used for the meta-analysis. In studies where change scores were missing, the following equation was used for calculating the change in mean and standard deviation (SD) scores: i) Mean (change) = mean (after)-mean (before); ii) SD (change) $=$ square root $\left\{\left[\mathrm{SD}^{2}\right.\right.$ (after) $-\mathrm{SD}^{2}$ (before) $\left.] / 2\right\}$.

Considering the heterogeneity amongst studies, a random-effects model was used to calculate the pooled effect size. The mean difference (SMD) with $95 \%$ confidence interval (CI) was used for combining the data. Heterogeneity was calculated using the $\mathrm{I}^{2}$ statistic. $\mathrm{I}^{2}$ values of $25-50 \%$ represented low, values of $50-75 \%$ medium and $>75 \%$ represented substantial heterogeneity.

\section{Results}

A total of 792 studies were screened by their abstracts (Fig. 1). Out of the 12 studies retrieved for full-text analysis, 5 were excluded. The reasons for exclusion were as follows: The use of non-invasive ventilation in the control group (19), the use of supplementary oxygen during home activities (20), crossover trial (21), the absence of a control group (22), and trials on pre-tested subjects showing improvement with supplemental oxygen (23). A total of 7 trials were included in this systematic review and meta-analysis (11-13,15,24-26).

Characteristics of the included studies. The details of studies included in the review are presented in Table II. The sample size was $<20$ patients per group in all studies, apart from 1 (25). Only 1 trial had a large sample of 58 patients in the study and 53 patients in the control group (25). Room air was used in the control group in 1 trial (11), while compressed/humidified air was used in the remaining studies. The exercise protocol included cycle ergometry in 3 studies $(15,24,26)$ and treadmill walking in 1 trial (13). Physical training was carried out in 3 sessions/week in all studies apart from 1 study (11), which utilized a 5 session/week protocol. The duration of each session varied from $30 \mathrm{~min}$ to $1 \mathrm{~h}$. In 1 trial, utilizing a 24-week PR program, outcomes were assessed at 12 and 24 weeks. To maintain homogeneity with the remaining studies, which conducted a 6-10 week PR program, outcome data of 12 weeks from this trial were included (26).

Outcomes and data analysis. The outcomes assessed varied across studies. Details of the outcome variables and conclusions of individual studies are presented in Table III.

Maximal exercise capacity. Maximal exercise capacity was tested by cycle ergometry in 4 trials $(11,15,24,26)$. The data of 55 participants in the study group and 54 in the control group were pooled for a meta-analysis. No statistically significant difference was found between the 2 groups in terms of power (random: $\mathrm{MD}=-2.38 ; 95 \% \mathrm{CI},-5.79$ to $1.03 ; \mathrm{P}=0.86, \mathrm{I}^{2}=0 \%$ ) 
(Fig. 2). Similarly, the maximum energy expenditure $\left(\mathrm{VO}_{2} \max \right)$ did not differ significantly between the supplemental oxygen and control groups (random: $\mathrm{MD}=-0.01 ; 95 \% \mathrm{CI},-0.06$ to $0.07 ; \mathrm{P}=0.45, \mathrm{I}^{2}=0 \%$ ) (Fig. 3). End-of test dyspnea scores were pooled from 3 trials $(11,15,24)$. The results of the meta-analysis indicated no significant advantage of supplemental oxygen in reducing dyspnea scores (random: $\mathrm{MD}=0.79 ; 95 \% \mathrm{CI}, 0.23$ to 1.36; $\mathrm{P}=0.51, \mathrm{I}^{2}=0 \%$ ) (Fig. 4). End-of test oxygen saturation $(\mathrm{SpO} 2 \%)$ was measured by 2 studies $(11,15)$. Both reported no advantage of supplemental oxygen.

Constant power exercise capacity. Three trials $(11,15,24)$ used cycle ergometry to measure constant power exercise capacity. Data were not homogenous to be pooled for a meta-analysis. Endurance capacity, measured as an increase in exercise time, increased only in the oxygen group in 1 study (11) (the change was not significant), while in another trial (15), it improved significantly in both groups. Isotime $\mathrm{VO}_{2}$ was recorded by 2 trials $(15,24)$. Both found no significant difference between the 2 groups. One trial (15) found end-of test dyspnea scores to be significantly improved in the oxygen group as compared to the control group. End-of test oxygen saturation $(\mathrm{SpO} 2 \%)$, measured by 2 trials $(11,15)$, did not differ significantly between the 2 groups.

Functional exercise capacity. Three studies $(11,13,26)$ measured functional exercise capacity using 6-min walk tests (6MWT). Pooled data of 41 participants in the study group and 39 in the control group indicated no significant differences between the 2 groups (random: $\mathrm{MD}=-14.93 ; 95 \% \mathrm{CI},-32.64$ to 2.78; $\mathrm{P}=0.93, \mathrm{I}^{2}=0 \%$ ) (Fig. 5). Two trials $(11,13)$ recorded end-of test dyspnea scores and end-of test oxygen saturation and found no significant difference between the supplemental oxygen and control groups.

Shuttle walk tests. Incremental shuttle walk tests were used as an outcome measure in 2 trials $(12,25)$. While 1 study (25) found no significant difference between groups, the other study recorded a significant reduction in end-of test dyspnea scores in the oxygen group (12). Data from the endurance shuttle walk test from 1 trial (25) demonstrated significant benefits in both groups with no significant in-between group difference.

Quality of life. Health-related quality of life was measured using the Chronic Respiratory Disease Questionnaire (CRQ) in 4 studies $(11,12,15,25)$. Data of 95 participants in the study group and 91 participants in the control group were analyzed. The results of meta-analysis of total scores indicated no difference between the 2 groups (random: $\mathrm{MD}=-0.09 ; 95 \% \mathrm{CI}$, -0.16 to $-0.01 ; \mathrm{P}=0.59, \mathrm{I}^{2}=0 \%$ ) (Fig. 6 ).

Quality of included studies. The authors' judgment of risk of bias of included trials is presented in Fig. 7. A total of 6 of the 7 studies described an adequate randomization method (11-13,15,25,26). Adequate information on allocation concealment was reported in 3 studies $(11,12,25)$. The blinding of participants and outcome assessment was clearly reported in 4 trials $(15,24-26)$. One trial had a significant number of drop-outs from the study (26).

\section{Discussion}

The primary objective of this systematic review and meta-analysis was to analyze the beneficial effect of supplemental oxygen during exercise training in patients with 


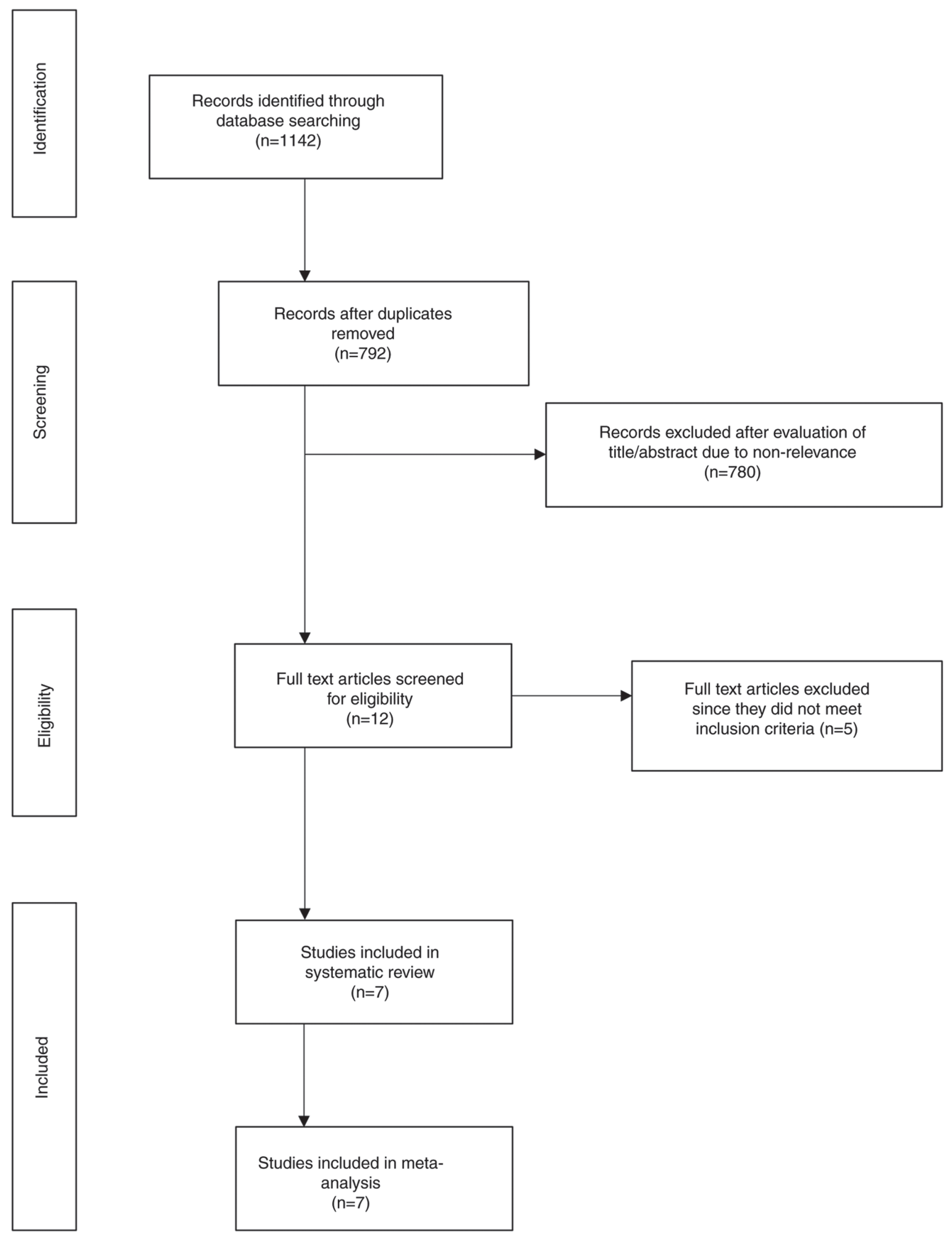

Figure 1. Search results of the study.

COPD. Overall, the results of this review can be categorized into 3 sub-headings: Exercise capacity, dyspnea scores and health-related quality of life.

Exercise tolerance is an important predictor of morbidity and mortality in COPD patients. A number of exercise testing protocols were utilized by the included studies. While maximal exercise testing measures the participants' response to a gradually increasing workload, a constant power exercise test assesses the participants systemic response to a constant metabolic demand (27). Since maximal exercise capacity is seldom reached during daily activities, the metabolic load of day-to-day activities is more suitably assessed using the constant power exercise test or other functional exercise tests like 6 or 12 MWT and shuttle walk tests (28). These investigations are also more sensitive to changes following intervention for exercise training (2). Another disadvantage of a maximal 


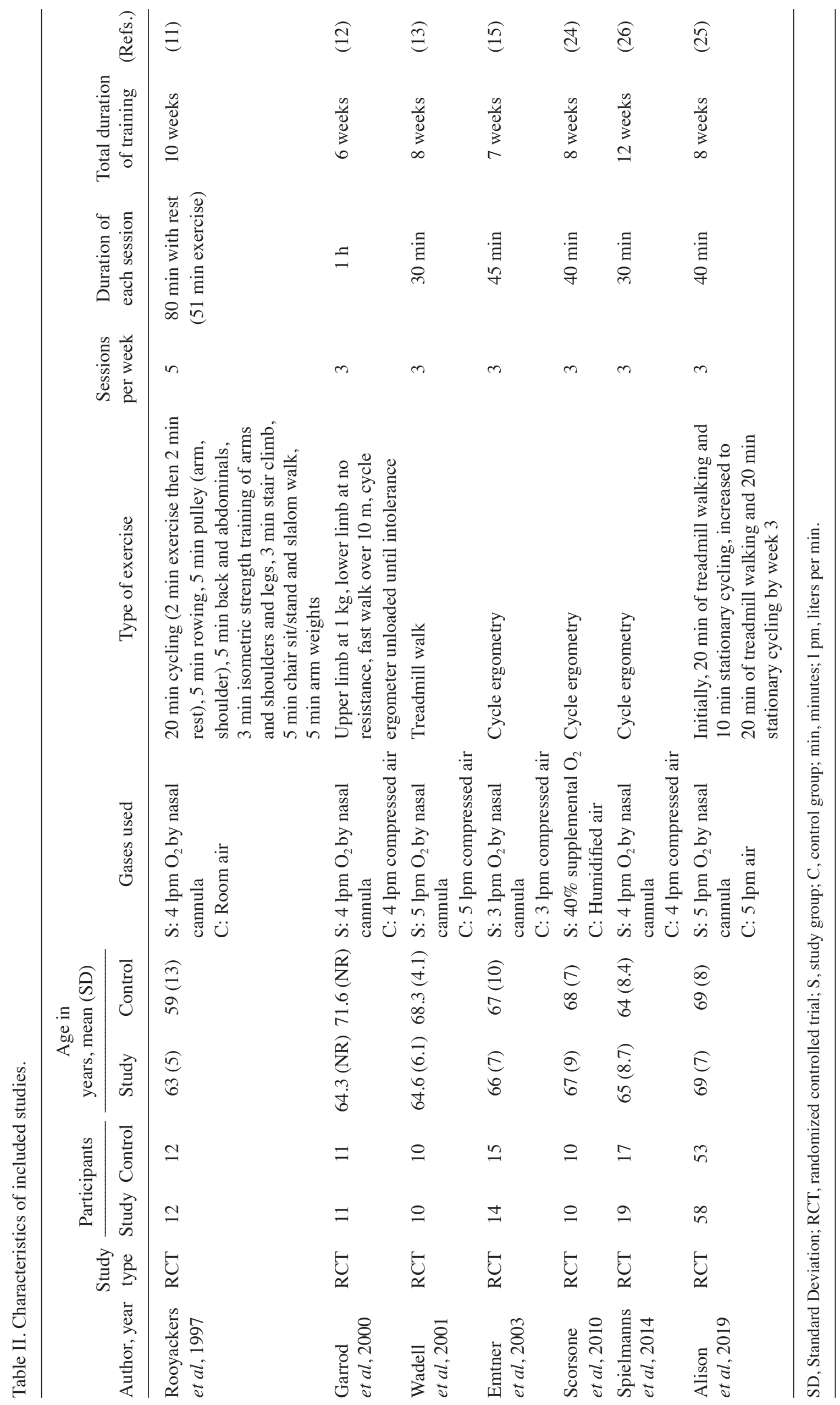


Table III. Outcomes of included studies.

\begin{tabular}{ll}
\hline Author, year & \multicolumn{1}{c}{ Outcomes assessed } \\
\hline Rooyackers & i) Maximal cycle ergometry-4 lpm $\mathrm{O}_{2}$ and room air \\
et al, 1997 & ii) Constant power cycle ergometry-30\% $\mathrm{O}_{2}$ and \\
& room air \\
& $\begin{array}{l}\text { iii) 6MWT-4 lpm } \mathrm{O}_{2} \text { and room air } \\
\text { iv) Stair climb-up 4, plateau, down } 3\end{array}$ \\
& v) Weight lift-lift between racks \\
& vi) Chronic respiratory Disease Questionnaire \\
& carbon monoxide
\end{tabular}

$\begin{array}{ll}\text { Garrod } & \text { i) Shuttle walk test } \\ \text { et al,2000 } & \text { ii) Chronic respiratory Disease Questionnaire } \\ & \text { iii) Hospital Anxiety and Depression Scale } \\ & \text { iv) London Chest Activity of Daily Living Scale }\end{array}$

Wadell i) $6 \mathrm{MWT}$ on $5 \mathrm{lpm} \mathrm{O}_{2}$ and $5 \mathrm{lpm}$ compressed et al, 2001 air (random order) with a $1 \mathrm{~h}$ rest in between ii) Arterial blood gas analysis

Emtner i) Maximal cycle ergometry-30\% $\mathrm{O}_{2}$ and et al, 2003 compressed air

ii) Constant power cycle ergometry-30\% $\mathrm{O}_{2}$ and compressed air

iii) Chronic respiratory disease questionnaire

iv) $\mathrm{SF}-36$

v) PFT-spirometry and lung volume

vi) Arterial blood gas analysis

Scorsone

i) PFT

et al, 2010

ii) Maximal cycle ergometry

iii) Constant power cycle ergometry

iv) Arterial blood gas analysis

Spielmanns i) 6MWT-room air

et al, 2014 ii) Maximal cycle ergometry-room air

iii) SF-36
Alison
i) Endurance shuttle walk test
et al, 2019
ii) Incremental shuttle walk test
iii) Chronic Respiratory Disease Questionnaire
iv) Dyspnoea-12 Questionnaire

Conclusions

(Refs.)

Pulmonary rehabilitation improved exercise

performance and quality of life in both groups. $\mathrm{O}_{2}$ supplementation during the training did not add to the effects of training on room air.

Supplemental $\mathrm{O}_{2}$ during training does little to enhance exercise tolerance although there is a small benefit in terms of dyspnea. Patients with severe disabling dyspnea may find symptomatic relief with supplemental oxygen.

Supplemental $\mathrm{O}_{2}$ did not further improve the training effect, compared with training with air, in patients with chronic obstructive pulmonary disease.

Supplemental $\mathrm{O}_{2}$ provided during high-intensity training yields higher training intensity and evidence of gains in exercise tolerance in laboratory testing.

$\mathrm{O}_{2}$ supplementation does not contribute to improved exercise performance in patients with moderate to severe COPD.

$\mathrm{O}_{2}$ supplemental oxygen during the training program had no additional benefits in improving quality of life and exercise capacity in subjects with moderate-to-severe COPD.

Both $\mathrm{O}_{2}$ and Air groups significantly improved exercise capacity and health related quality of life with no greater benefit from training with supplemental $\mathrm{O}_{2}$ than with medical air.

lpm, liter per minute; 6MWT, 6-min walking distance test; SF-36, Medical Outcomes Survey Short Form 36 questionnaire; PFT, Pulmonary Function Test.

exercise test is the high level of motivation required on behalf of the participant to achieve the actual maximal limit. Secondly, approximately 6-10\% of day-to-day variation in $\mathrm{VO}_{2}$ max can be observed in patients with COPD (29). Maximal exercise test using cycle ergometry was utilized by 4 of the 7 included trials. On pooling of the data, supplemental oxygen during exercise training was not found to be beneficial in improving $\mathrm{VO}_{2}$ max and power output.
In the absence of homogenous data, quantitative analysis was not carried out of the 3 trials reporting constant power exercise tests. The results of endurance capacity, measured as an increase in exercise time wer ambiguous. Emtner et al (15), while reporting a significant improvement in exercise time in both groups, reported better outcomes in the supplemental oxygen group. The authors suggested that the results may have been underestimated as 16 tests in the 


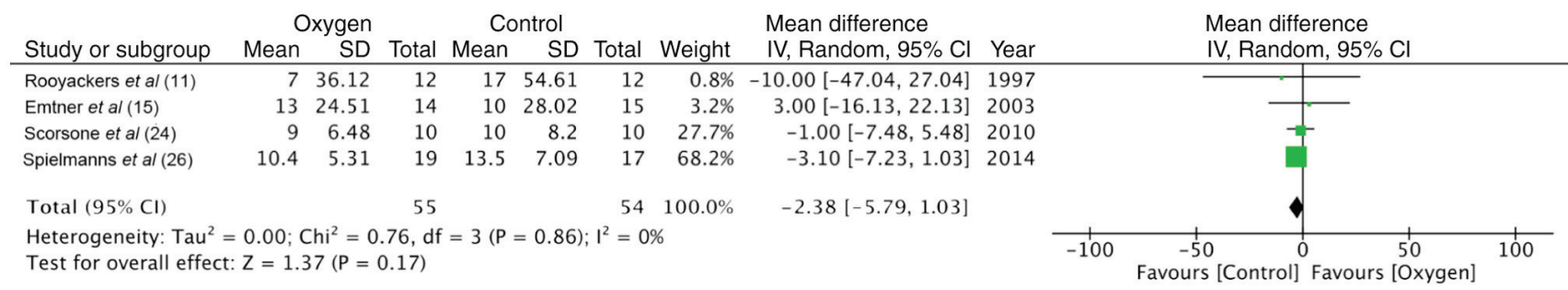

Figure 2. Forrest plot of oxygen vs. control for power outcome of maximal exercise test.

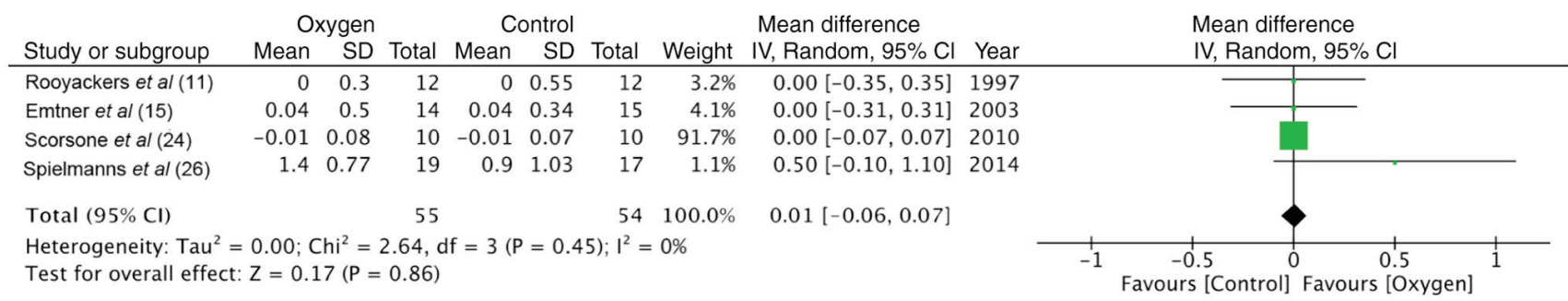

Figure 3. Forrest plot of oxygen vs. control for $\mathrm{VO}_{2}$ outcome of maximal exercise test.

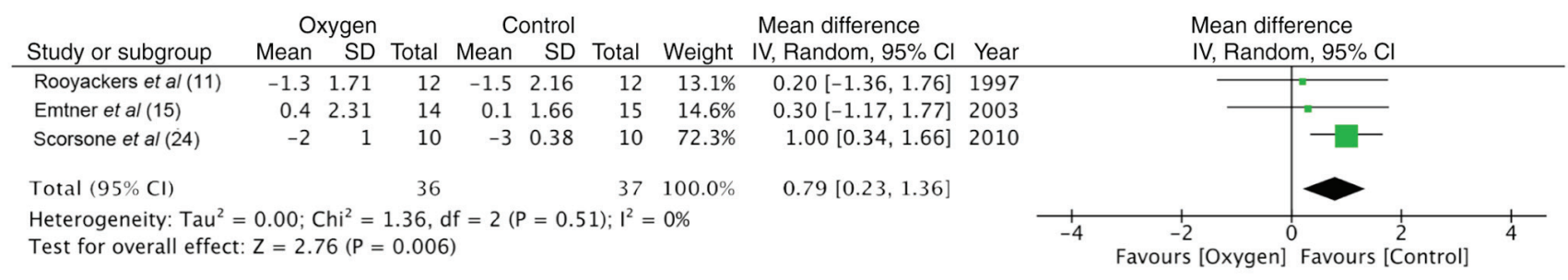

Figure 4. Forrest plot of oxygen vs. control for end-of test dyspnea scores of maximal exercise test.

\begin{tabular}{|c|c|c|c|c|c|c|c|c|c|c|c|c|}
\hline \multirow[b]{2}{*}{ Study or subgroup } & \multicolumn{3}{|c|}{ Oxygen } & \multicolumn{3}{|c|}{ Control } & \multicolumn{3}{|c|}{ Mean difference } & \multirow{2}{*}{\multicolumn{3}{|c|}{$\begin{array}{l}\text { Mean difference } \\
\text { IV, Random, } 95 \% \mathrm{Cl}\end{array}$}} \\
\hline & Mean & SD & Total & Mean & SD & Total & Weight & IV, Random, $95 \% \mathrm{Cl}$ & Year & & & \\
\hline Rooyackers et al (11) & 86 & 161.25 & 12 & 123 & 178.94 & 12 & $1.7 \%$ & $-37.00[-173.29,99.29]$ & 1997 & & t & \\
\hline Wadell et al (13) & 31 & 79.03 & 10 & 52 & 65.62 & 10 & $7.7 \%$ & $-21.00[-84.67,42.67]$ & 2001 & & & \\
\hline Spielmanns et al (26) & 56 & 20.97 & 19 & 70 & 33.76 & 17 & $90.6 \%$ & $-14.00[-32.61,4.61]$ & 2014 & & & \\
\hline Total $(95 \% \mathrm{Cl})$ & & & 41 & & & 39 & $100.0 \%$ & $-14.93[-32.64,2.78]$ & & & t & \\
\hline $\begin{array}{l}\text { Heterogeneity: } \text { Tau }^{2} \\
\text { Test for overall effect }\end{array}$ & $\begin{array}{l}0.00 \\
z=1.6\end{array}$ & $\begin{array}{l}-h i^{2}=0 \\
55(P=0\end{array}$ & $\begin{array}{l}\text { 15, df }= \\
.10)\end{array}$ & & .93); & & & & & -1000 & $\begin{array}{ccc}-500 & 0 & 500 \\
\text { Favours [Control] Favours [Oxygen] }\end{array}$ & 1000 \\
\hline
\end{tabular}

Figure 5. Forrest plot of oxygen vs. control for 6-min walk tests.

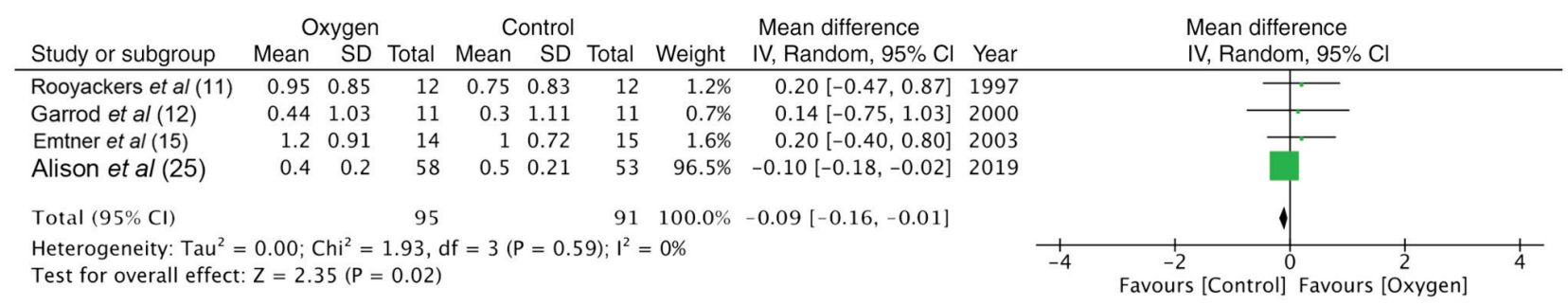

Figure 6. Forrest plot of oxygen vs. control for quality of life outcome.

oxygen-trained group were terminated at 30 min versus only 7 tests in the control group. Rooyackers et al (11) found an increase in the cycling time only in the experimental group with no change in the control group; however, the change not was statistically significant. Outcome measurements at specific time-points (isotime responses) during constant power exercise tests are effort-independent and allow for a more robust comparison of intervention effects (30). While our review indicates no significant change in $\mathrm{VO}_{2}$ max and cardiovascular parameters (heart rate and blood pressure) 


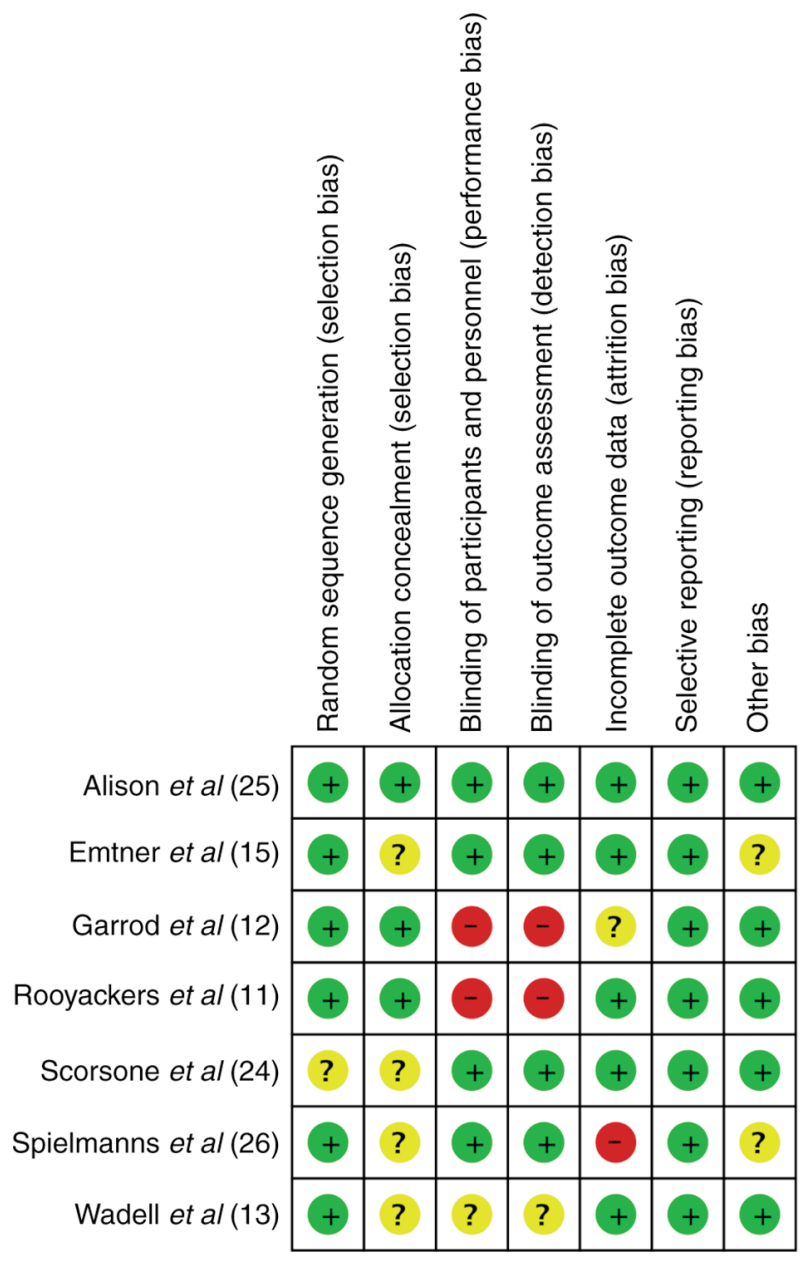

Figure 7. Risk of bias summary.

with supplemental oxygen therapy, Emtner et al (15) documented a slower and deeper pattern of breathing in the oxygen-trained group.

Non-laboratory functional exercise tests, such as 6MWT have been validated to assess the functional status of patients with COPD (31). Not only are they easy to perform and inexpensive, but standardization is also easy for measuring the effect of interventions. The results from the meta-analysis of 6MWT suggest no benefits with the use of supplemental oxygen during exercise training of patients with COPD. Similar results were found by Garrod et al (12) and Alison et al (25) during shuttle walk tests. Although some authors (13) have reported better performance of the oxygen group during training sessions, the outcome assessment on room air failed to demonstrate any benefit despite prolonged training. One possible explanation suggested is that control groups train under slight hypoxemia as compared to the oxygen groups. The 'slight hypoxemia' causes physiological stress, which is required for improving physical capacity (13).

Dyspnea is an important symptom that limits exercise tolerance in patients with COPD. The actual role of oxygen in improving dyspnea is complex and not yet well understood (32). Dyspnea was recorded as an outcome variable in majority trials of this review. However, meta-analysis for dyspnea scores measured on the Borg scale was conducted only for the maximal exercise test, which demonstrated no benefit of supplemental oxygen therapy. Garrod et al (12) demonstrated a reduction in end-of test dyspnea scores after shuttle walk tests but without any significant change of other variables like exercise capacity and health-related quality of life outcomes. The isolated dyspnea results may be attributed to the small sample size of the study.

PR has been shown to significantly improve quality of life outcomes in patients with COPD (7). PR programs include an exercise module and an education/psychological support module. While the exact contribution of each module in improving quality of life is unknown, a recent review suggested that self-management educational programs can significantly improve the quality of life of COPD patients (33). Health-related quality of life outcome was measured via CRQ in 4 studies $(11,12,15,25)$ included in the review. Our analysis failed to demonstrate any beneficial effect of supplemental oxygen in improving the quality of life of patients with COPD.

Some limitations of our study need to be elaborated. The strength of any systematic review and meta-analysis depends upon the homogeneity and quality of the included studies. The lack of homogeneity of the included trials is the largest drawback of this study. There was a wide variation in the methodology, intervention, exercise protocol and outcome measurements, which precluded a quantitative analysis for all variables. The inclusion criteria also varied between studies. While majority trials were carried out on participants who desaturate on exercise, studies by Emtner et al (15) and Spielmanns et al (26) focused only on patients with COPD who were normoxemic at rest and exercise. A sub-group analysis analyzing the effect of oxygen on each group was not possible considering the heterogeneity. Secondly, not all studies reported adequate allocation concealment with blinding of participants and outcome assessment. A lack of rigorous methodology could have affected results. Thirdly, the sample size of majority studies was small with less than 20 participants per group.

Nevertheless, this study is an important update over the previous review on this topic (2). Our results indicate that supplemental oxygen during the exercise training of patients with COPD does not improve exercise capacity, dyspnea scores and quality of life. However, the quality of the evidence is weak. Multi-center RCTs with homogenous methodology and intervention are required to provide stronger evidence on this subject.

\section{Acknowledgements}

Not applicable.

\section{Funding}

No funding was received.

\section{Availability of data and materials}

All data generated or analyzed during this study are included in this published article or are available from the corresponding author on reasonable request. 


\section{Authors' contributions}

YL and FG designed and searched literature. YL prepared the manuscript. FG edited the manuscript. Both authors have read and approved the final manuscript.

\section{Ethics approval and consent to participate}

Not applicable.

\section{Patient consent for publication}

Not applicable.

\section{Competing interests}

The authors declare that they have no competing interests.

\section{References}

1. Celli BR and MacNee W; ATS/ERS Task Force: Standards for the diagnosis and treatment of patients with COPD: A summary of the ATS/ERS position paper. Eur Respir J 23: 932-946, 2004.

2. Nonoyama ML, Brooks D, Lacasse Y, Guyatt GH and Goldstein RS: Oxygen therapy during exercise training in chronic obstructive pulmonary disease. Cochrane Database Syst Rev 2: CD005372, 2007.

3. Ejiofor SI, Bayliss S, Gassamma A and Turner AM: Ambulatory oxygen for exercise-induced desaturation and dyspnea in chronic obstructive pulmonary disease (COPD): Systematic review and meta-analysis. Chronic Obstr Pulm Dis 3: 419-434, 2016.

4. Wagner PD: Skeletal muscles in chronic obstructive pulmonary disease: Deconditioning, or myopathy? Respirology 11: 681-686, 2006.

5. Spruit MA, Singh SJ, Garvey C, ZuWallack R, Nici L, Rochester C, Hill K, Holland AE, Lareau SC, Man WD, et al: An official American Thoracic Society/European Respiratory Society statement: Key concepts and advances in pulmonary rehabilitation. Am J Respir Crit Care Med 188: e13-e64, 2013.

6. Ries AL, Bauldoff GS, Carlin BW, Casaburi R, Emery CF, Mahler DA, Make B, Rochester CL, Zuwallack R and Herrerias C: Pulmonary rehabilitation: Joint ACCP/AACVPR evidence-based clinical practice guidelines. Chest 131 (5 Suppl): 4S-42S, 2007.

7. McCarthy B, Casey D, Devane D, Murphy K, Murphy E and Lacasse Y: Pulmonary rehabilitation for chronic obstructive pulmonary disease. Cochrane Database Syst Rev 2: CD003793, 2015.

8. Henriksson J and Reitman JS: Quantitative measures of enzyme activities in type I and type II muscle fibres of man after training. Acta Physiol Scand 97: 392-397, 1976.

9. Cranston JM, Crockett A, Moss J and Alpers JH: Domiciliary oxygen for chronic obstructive pulmonary disease. Cochrane Database Syst Rev CD001744, 2005.

10. Jolly EC, Di Boscio V, Aguirre L, Luna CM, Berensztein S and Gené RJ: Effects of supplemental oxygen during activity in patients with advanced COPD without severe resting hypoxemia. Chest 120: 437-443, 2001.

11. Rooyackers JM, Dekhuijzen PN, Van Herwaarden CL and Folgering HT: Training with supplemental oxygen in patients with COPD and hypoxaemia at peak exercise. Eur Respir J 10 1278-1284, 1997.

12. Garrod R, Paul EA and Wedzicha JA: Supplemental oxygen during pulmonary rehabilitation in patients with COPD with exercise hypoxaemia. Thorax 55: 539-543, 2000.

13. Wadell K, Henriksson-Larsén K and Lundgren R: Physical training with and without oxygen in patients with chronic obstructive pulmonary disease and exercise-induced hypoxaemia. J Rehabil Med 33: 200-205, 2001.

14. Pépin JL and Lévy P: Principles of oxygen therapy. In: Respiratory Medicine. Gibson G, Geddes D, Costabel U, Sterk P and Corrin B (eds). 3rd edition. Saunders, Edinburgh, pp502-521, 2003.
15. Emtner M, Porszasz J, Burns M, Somfay A and Casaburi R: Benefits of supplemental oxygen in exercise training in nonhypoxemic chronic obstructive pulmonary disease patients. Am J Respir Crit Care Med 168: 1034-1042, 2003.

16. Moher D, Liberati A, Tetzlaff J, Altman DG and PRISMA Group: Preferred reporting items for systematic reviews and meta-analyses: The PRISMA statement. PLoS Med 6: e1000097, 2009.

17. Higgins J and Green S (eds): Cochrane Handbook for Systemic Reviews of Interventions. Version 5.1.0. The Cochrane Collaboration, 2011. http://handbook.cochrane.org. Updated March 2011.

18. Higgins J, Altman D and Sterne J (eds): Chapter 8: Assessing risk of bias in included studies. In: Cochrane Handbook for Systemic Reviews of Interventions. Version 5.1.0. The Cochrane Collaboration, 2011. http://handbook.cochrane.org. Updated March 2011.

19. Borghi-Silva A, Mendes RG, Toledo AC, Malosá Sampaio LM, da Silva TP, Kunikushita LN, Dutra de Souza HC, Salvini TF and Costa D: Adjuncts to physical training of patients with severe COPD: Oxygen or noninvasive ventilation? Respir Care 55: 885-894, 2010.

20. Ringbaek T, Martinez G and Lange P: The long-term effect of ambulatory oxygen in normoxaemic COPD patients: A randomised study. Chron Respir Dis 10: 77-84, 2013.

21. Neunhäuserer D, Steidle-Kloc E, Weiss G, Kaiser B, Niederseer D, Hartl S, Tschentscher M, Egger A, Schönfelder M, Lamprecht B, et al: Supplemental oxygen during high-intensity exercise training in nonhypoxemic chronic obstructive pulmonary disease. Am J Med 129: 1185-1193, 2016.

22. Helgerud J, Bjørgen S, Karlsen T, Husby VS, Steinshamn S, Richardson RS and Hoff J: Hyperoxic interval training in chronic obstructive pulmonary disease patients with oxygen desaturation at peak exercise. Scand J Med Sci Sports 20: e170-e176, 2010.

23. Dyer F, Callaghan J, Cheema K and Bott J: Ambulatory oxygen improves the effectiveness of pulmonary rehabilitation in selected patients with chronic obstructive pulmonary disease. Chron Respir Dis 9: 83-91, 2012.

24. Scorsone D, Bartolini S, Saporiti R, Braido F, Baroffio M, Pellegrino R, Brusasco V and Crimi E: Does a low-density gas mixture or oxygen supplementation improve exercise training in COPD? Chest 138: 1133-1139, 2010.

25. Alison JA, McKeough ZJ, Leung RWM, Holland AE, Hill K, Morris NR, Jenkins S, Spencer LM, Hill CJ, Lee AL, et al: Oxygen compared to air during exercise training in COPD with exercise-induced desaturation. Eur Respir J 53: 1802429, 2019.

26. Spielmanns M, Fuchs-Bergsma C, Winkler A, Fox G, Krüger S and Baum K: Effects of oxygen supply during training on subjects with COPD who are normoxemic at rest and during exercise: A blinded randomized controlled trial. Respir Care 60: 540-548, 2015.

27. Oga T, Nishimura K, Tsukino M, Hajiro T, Ikeda A and Izumi T: The effects of oxitropium bromide on exercise performance in patients with stable chronic obstructive pulmonary disease. Am J Respir Crit Care Med 161: 1897-1901, 2000.

28. Singh SJ, Morgan MD, Hardman AE, Rowe C and Bardsley PA: Comparison of oxygen uptake during a conventional treadmill test and the shuttle walking test in chronic airflow limitation. Eur Respir J 7: 2016-2020, 1994.

29. Noonan V and Dean E: Submaximal exercise testing: Clinical application and interpretation. Phys Ther 80: 782-807, 2000.

30. Nicolò A, Bazzucchi I, Haxhi J, Felici F and Sacchetti M: Comparing continuous and intermittent exercise: An 'isoeffort' and 'isotime' approach. PLoS One 9: e94990, 2014.

31. Lam HS, Lau FW, Chan GK and Sykes K: The validity and reliability of a 6-metre timed walk for the functional assessment of patients with stroke. Physiother Theory Pract 26: 251-255, 2010.

32. Sweer L and Zwillich CW: Dyspnea in the patient with chronic obstructive pulmonary disease. Etiology and management. Clin Chest Med 11: 417-445, 1990.

33. Wang T, Tan JY, Xiao LD and Deng R: Effectiveness of disease-specific self-management education on health outcomes in patients with chronic obstructive pulmonary disease: An updated systematic review and meta-analysis. Patient Educ Couns 100: 1432-1446, 2017.

This work is licensed under a Creative Commons Attribution-NonCommercial-NoDerivatives 4.0 International (CC BY-NC-ND 4.0) License. 